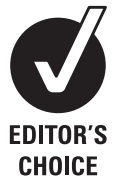

${ }^{1}$ The Ludwig Boltzmann Institute of Retinology and Biomicroscopic Laser surgery, Department of Ophthalmology, Rudolf Foundation Clinic, Vienna, Austria

${ }^{2}$ Biomedical Imaging Group, Department of Optometry and Vision Sciences, Cardiff University, Wales, UK

${ }^{3}$ Department of Medical Statistics, Core Unit of Medical Statistics and Informatics, Medical University of Vienna, Vienna, Austria

\section{Correspondence to} Dr Christiane I Falkner-Radler, The Ludwig Boltzmann Institute of Retinology and Biomicroscopic Lasersurgery, Department of Ophthalmology, Rudolf Foundation Clinic, Juchgasse 25, A 1030 Vienna, Austria; christiane.falkner-radler@ wienkav.at

Accepted 30 April 2010 Published Online First 7 July 2010

\title{
Human retinal pigment epithelium (RPE) transplantation: outcome after autologous RPE-choroid sheet and RPE cell-suspension in a randomised clinical study
}

\author{
Christiane I Falkner-Radler, ${ }^{1}$ Ilse Krebs, ${ }^{1}$ Carl Glittenberg, ${ }^{1}$ Boris Považay, ${ }^{2}$ \\ Wolfgang Drexler, ${ }^{2}$ Alexandra Graf, ${ }^{3}$ Susanne Binder ${ }^{1}$
}

\section{ABSTRACT}

Aims To evaluate the outcome after two types of retinal pigment epithelium (RPE) transplantation techniques.

Methods Fourteen consecutive patients with advanced exudative age-related macular degeneration (AMD) were randomly assigned to RPE-choroid sheet transplantation (group 1) or RPE cell-suspension transplantation (group 2). Outcome measures included best corrected distance and near visual acuity (BCVA), complication and recurrence rates, autofluorescence (AF), angiography, and time-domain and spectral-domain optical coherence tomography (TD- and SD-OCT).

Results A gain of three or more lines in BCVA at 24 months was found in two patients in group 1 and in one patient in group 2, whereas a loss of vision of three or more lines occurred in one patient in each group.

Revision surgery for proliferative vitreoretinopathy was required in one patient in group 1. Epiretinal membranes developed in two patients in group 1 and in one patient in group 2. No recurrence occurred in this series. AF showed hyperfluorescence coincident with the graft in group 1, and hyper- and hypofluorescence in irregular patterns in group 2. Revascularisation of the graft was present in all patients in group 1, and a normal choroidal vasculature in the area of RPE atrophy in all patients in group 2. OCT showed a decrease in retinal thickness in all patients, with an improved visualisation of inter- and intralaminar structures with SD-OCT.

Conclusion The anatomical and functional outcome after both RPE transplantation techniques was comparable. Intrastructural irregularities of the sheet assessed using SD-OCT might explain the rather limited visual gain in otherwise successful sheet transplants.

Clinical Trial Registration NCT00401713

\section{INTRODUCTION}

Despite the introduction of anti-vascular endothelial growth factor (VEGF) treatment resulting in significant improvement of vision in patients with neovascular age-related macular-degeneration (AMD), the treatment for advanced $\mathrm{AMD}$, geographic atrophy and/or non-responders is still controversial. Maculoplasty may be an alternative surgical approach in these patients, with the potential of restoring the retinal anatomy and achieving stable long-term results. ${ }^{1}$

Transplantation of retinal pigment epithelium (RPE) was introduced more than two decades ago, and considerable progress has been made regarding surgical technique and outcome..$^{2-4}$ In the clinical situation drawbacks as well as successes have been reported. ${ }^{5-9}$ Today, autologous RPE is used in clinical studies because homologous transplants make long-term combined immunosuppressive therapies necessary, and these are not well tolerated by elderly patients. ${ }^{10}$ Besides cell condition, basal lamina changes and cell survival, refinement of surgical technique is an important factor for improvement of anatomical and functional outcome. Two different transplantation techniques are currently used in clinical studies: the RPEsuspension technique and the RPE-choroid-sheet technique. ${ }^{11} 12$ The RPE-cell suspension, providing limited amounts of cells on a partially defective basal lamina can easily be delivered subretinally through small retinotomies, which can be sealed with a gas bubble. The translocation of a full thickness RPE-choroidal sheet, providing a regular cell sheet of polarised RPE on its own basal lamina carries a high risk for intra- and postoperative complications. Thus, a silicone oil tamponade is mandatory, necessitating a second vitreoretinal surgery for silicone oil removal.

This randomised clinical cohort study is the first study evaluating these two autologous RPE-transplantation techniques in advanced neovascular AMD.

\section{MATERIALS AND METHODS}

Consecutive patients with foveal choroidal neovascularisation (CNV) related to $\mathrm{AMD}$ were included in the study if they fulfilled all the following criteria: (1) age $>50$ years; (2) progression of the disease combined with progressive visual loss over the past 3 to 6 months; (3) not suitable for laser therapy and/or or photodynamic therapy (PDT) (large lesions, lesions containing $>50 \%$ pigment epithelium detachment (PED) or haemorrhage); and (4) 'non-responders' to the aforementioned therapies and to at least three, monthly performed, intravitreal anti-VEGF treatments. Non-responders were defined as patients presenting at least two of the following criteria: increase of lesion size (fluorescein angiography), increase of retinal thickness (RT), decrease of best corrected visual acuity (BCVA).

Patients were randomly assigned to receive either a full RPE-choroid sheet transplantation and silicone oil tamponade (group 1) or transplantation of an RPE-cell suspension combined with a gas 
tamponade (group 2). All surgical procedures were performed by one surgeon (SB). The principles of the surgical technique for the RPE-choroid graft were similar to the technique described by van Meurs et $a l^{7}$ After 20 gauge pars plana vitrectomy and/or simultaneous cataract surgery, the membrane removal was performed. Then perfluorocarbon (PFC) was injected, and a retinal quadrangle was surrounded with diathermy and/or intensive laser treatment before tissue preparation in the superior mid peripheral region of the retina. For this manoeuvre a switch from the non-contact wide-angle system (B.I.O.M. II; OCULUS, Wetzlar, Germany) to corneal lenses was performed, and a chandelier light was used. The PFC was removed, and a small amount of fluid was injected subretinally to provide delivery of the transplant with minimal trauma. The graft was gently grasped on its choroidal side and cut free. Then the retina on the flap was removed, and the RPE-choroid sheet was translocated subfoveally. After correct graft positioning, PFC was injected again, followed by a silicone-PFC fluid exchange as the last surgical step. Three to 6 months later, silicone oil removal was planned. Our technique for RPE-cell suspension has been described in detail previously. 814

Patients were examined before surgery and at 1 week, 1 month, and at 3, 9, 12 and 24 months after surgery. Clinical examinations included BCVA (Early Treatment Diabetic Retinopathy Study (ETDRS) charts), Radner reading charts (logarithm of reading acuity determination ( $\log R A D)$ ), biomicroscopy, autofluorescence, fluorescein and indocyanine green angiography (Heidelberg Engineering $\mathrm{GmbH}$, Heidelberg, Germany), and time-domain and spectral-domain optical coherence tomography (TD- and SD-OCT) scans. In addition, all patients underwent microperimetry at the 24 months followup using the MP-1 microperimeter (Nidek Technologies, Vigonza, Italy) with a Goldamm III and a 4-2 threshold algorithm. Two ophthalmologists uninvolved in the selection of the patients and surgery performed the examinations.

A masked statistical analysis was performed using SAS Software version 9.1 (SAS Institute Inc., North Carolina, USA) including univariate and stepwise regression analyses to find differences between both treatment groups and to determine the association between main outcome variables (best- corrected distance and near visual acuity (BCVA) and retinal thickness (RT)) at 24 months, baseline demographics and complication rates. Analyses of variance (ANOVA) were used to determine the association between baseline demographics and time course of BCVA and RT. A two-sided $p$ value less than 0.05 was considered statistically significant.

\section{RESULTS}

Fourteen patients who underwent surgery between November 2004 and October 2006 were included in this study. The surgery was uneventful in all patients. Simultaneous cataract surgery was performed in 10 patients; the other four patients were pseudophakic before surgery. No significant difference between the treatment groups and baseline demographics was found ( $p>0.05)$, shown in table 1 .

In group 1, a proliferative vitreoretinopathy (PVR) retinal detachment developed at 6 weeks after surgery in one patient. Revision surgery consisted of silicone oil removal, re-vitrectomy, membrane peeling, laser treatment and silicone oil tamponade because of a residual retinal detachment nasally superior. Due to massive membrane formation, silicone oil removal was not possible in this patient. All other patients underwent silicone oil removal, and in two patients a simultaneous removal of preretinal membranes was performed. In group 2, a preretinal membrane developed in one patient, but it was not surgically removed because of the patient's low vision. No significant difference for complication rates was found between both treatment groups $(p=0.56)$. No recurrence was encountered in this case series.

In group 1, two patients showed a gain of 3 or more lines in distance BCVA at 24 months. Another patient showed a loss of 3 or more lines at 24 months, and four patients showed stable vision within 2 lines. The two patients with significant improvement in distance acuity regained reading ability during follow-up. Two other patients, one presented with a PVR retinal detachment and a significant loss of distance BCVA, lost reading ability during follow-up. Another patient did not recover reading vision after surgery. In group 2, one patient showed a gain of 3 or more lines in distance BCVA at 24 months, and another patient had a loss of 3 or more lines after surgery. The other five patients presented with stable vision within 2 lines. One patient in this group regained reading ability during follow-up, another patient lost reading vision after surgery, and two other patients did not recover any reading ability at 24 months. No significant difference for distance and near BCVA was found between both treatment groups $(p>0.75)$. In addition, baseline demographics were not significantly associated with the visual outcome in both groups ( $p>0.05)$. Details on BCVA are presented in table 2 , and in figure 1 and 2 .

In group 1, autofluorescence showed hyperfluorescent areas of different intensity corresponding to the RPE-choroid sheet in all cases. These findings showed some decrease in size and loss of intensity up to normal fluorescence during follow-up (figure 3c,d). In group 2, autofluorescence showed areas of hypo- and hyperfluorescence in irregular patterns and well demarked areas of RPE atrophy with absent autofluorescence, remaining similar in size and shape during follow-up (figure 4f). Two cases showed small areas of normal autofluorescence within the area of RPE atrophy. In group 1, revascularisation of the graft was visible on fluorescein and indocyanine green angiography in all cases, but did not correspond to AF of the RPE

Table 1 Patients' demographic data undergoing two different autologous retinal pigment epithelium (RPE) transplantation techniques

\begin{tabular}{|c|c|c|c|}
\hline & $\begin{array}{l}\text { RPE-choroid } \\
\text { sheet TX } \\
\text { (group 1) }\end{array}$ & $\begin{array}{l}\text { RPE cell-s } \\
\text { uspension TX } \\
\text { (group 2) }\end{array}$ & $\begin{array}{l}p \\
\text { Value }\end{array}$ \\
\hline Patients (n) & 7 & 7 & \\
\hline Mean age (range, years) & $77(72-82)$ & $79(71-84)$ & 0.35 \\
\hline Sex & $\begin{array}{l}5 \text { women, } \\
2 \text { men }\end{array}$ & $\begin{array}{l}4 \text { women, } \\
3 \text { men }\end{array}$ & 1.0 \\
\hline Mean lesion size (range, $\mathrm{mm}$ ) & $7.8(3.5-10)$ & $6.1(4.9-9.2)$ & 1.0 \\
\hline Diagnosis (n) & & & 0.59 \\
\hline Classic CNV with haemorrhage & 5 & 3 & \\
\hline Occult CNV, occult and classic CNV & 2 & 4 & \\
\hline Non-responder to previous therapy (n) & 5 & 2 & 0.29 \\
\hline $\begin{array}{l}\text { Distance BCVA (mean } \pm S D \text {, } \\
\text { ETDRS chart) }\end{array}$ & $0.25 \pm 0.22$ & $0.28 \pm 0.23$ & 0.75 \\
\hline Range & $\begin{array}{l}\mathrm{LP}(\mathrm{n}=2) \\
\text { to } 0.55\end{array}$ & $0.04-0.53$ & \\
\hline Near BCVA (mean $\pm S D$, logRAD) & $1.3 \pm 0.7$ & $1.3 \pm 0.7$ & 0.79 \\
\hline Range & $\begin{array}{l}\text { NRA }(n=2) \\
\text { to } 0.6\end{array}$ & $\begin{array}{l}\text { NRA }(n=2) \\
\text { to } 0.4\end{array}$ & \\
\hline Mean RT (range, $\mu \mathrm{m}$ ) & $565(398-750)$ & $482(464-498)$ & 0.54 \\
\hline Mean follow-up time (range, months) & $27(24-36)$ & $32(24-42)$ & \\
\hline
\end{tabular}

$\mathrm{p}<0.05$ (statistically significant).

BCVA, best corrected distance and near visual acuity; CNV, choroidal neovascularisation; ETDRS, Early Treatment Diabetic Retinopathy Study; logRAD, logarithm of reading acuity determination; LP, light perception; NRA, no reading ability; RPE, retinal pigment epithelium; $\mathrm{RT}$, retinal thickness; TX, transplantation. 
Table 2 Functional outcome after retinal pigment epithelium (RPE) transplantation

\begin{tabular}{lcl}
\hline Patients included & $\begin{array}{c}\text { RPE-choroid sheet } \\
\text { TX (group 1) }(\mathbf{n}=\mathbf{7})\end{array}$ & $\begin{array}{l}\text { RPE cell-suspension } \\
\text { TX (group 2) }(\mathbf{n}=\mathbf{7})\end{array}$ \\
\hline $\begin{array}{l}\text { Distance BCVA (mean } \pm S D, \\
1 \text { ETDRS chart) }\end{array}$ \\
month & $0.11 \pm 0.15$ & $0.07 \pm 0.10$ \\
3 months & $0.11 \pm 0.12$ & $0.07 \pm 0.08$ \\
9 months & $0.10 \pm 0.13$ & $0.12 \pm 0.15$ \\
12 months & $0.13 \pm 0.20$ & $0.16 \pm 0.15$ \\
24 months & $0.20 \pm 0.16$ & $0.26 \pm 0.17$ \\
Near BCVA (mean $\pm S D, ~ l o g R A D)$ & \\
1 month & $1.8 \pm 0.3$ & $1.8 \pm 0.3$ \\
3 months & $1.6 \pm 0.4$ & $1.7 \pm 0.3$ \\
9 months & $1.6 \pm 0.5$ & $1.7 \pm 0.3$ \\
12 months & $1.5 \pm 0.4$ & $1.7 \pm 0.3$ \\
24 months & $1.5 \pm 0.4$ & $1.6 \pm 0.3$ \\
\hline
\end{tabular}

BCVA, best corrected distance and near visual acuity; logRAD, logarithm of reading acuity determination; RPE, retinal pigment epithelium; TX, transplantation.

(figure $3 e, f, g, h)$. In group 2 , hyperfluorescence (window defect) was present on fluorescein angiography (figure 4g) and a normal choroidal vasculature on indocyanine green angiography (figure $4 \mathrm{~h}$ ) in the area of RPE atrophy in all cases. In addition, visual acuity did not correlate with intensity of AF.

OCT measurements showed a decrease in RT after surgery in all patients in this case series. In group 1, mean RT at baseline was $565 \mu \mathrm{m}$ and decreased to $415 \mu \mathrm{m}$ at 24 months. In group 2, mean RT at baseline was $482 \mu \mathrm{m}$ and decreased to $322 \mu \mathrm{m}$ at 24 months. With TD-OCT the RPE-choroid sheet appeared well placed and flat in the subretinal area, while with SD-OCT intralaminal and intrastructural abnormalities were visible within the graft. In the area of atrophy surrounding the graft an enhanced permeability into the choroid was present. This increased permeability was absent in the area of the graft. After RPE cell-suspension, localised increased permeability into the tissue in areas of RPE atrophy was more clearly visualised with SD-OCT than TD-OCT. In both groups, SD-OCT showed

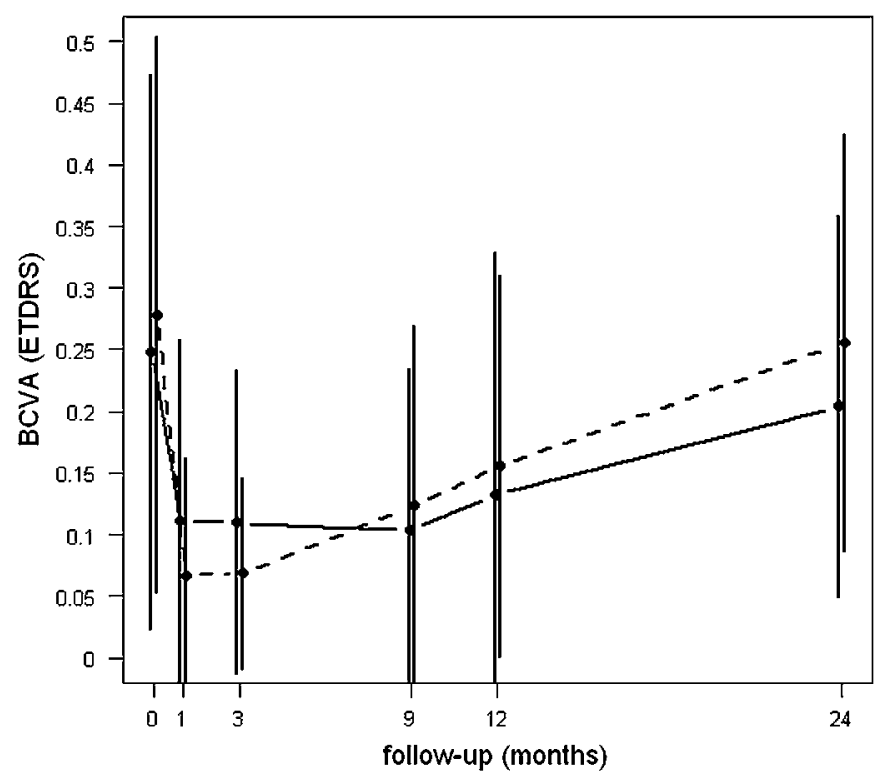

Figure 1 Mean values (points) and standard deviations (vertical lines) of distance best-corrected visual acuity (BCVA) measurements using Early Treatment Diabetic Retinopathy Study (ETDRS) charts. Charts over time are shown for treatment group 1 (retinal pigment epithelium (RPE)choroid sheet transplantation, solid line) and treatment group 2 (RPE-cell suspension transplantation, dashed line).

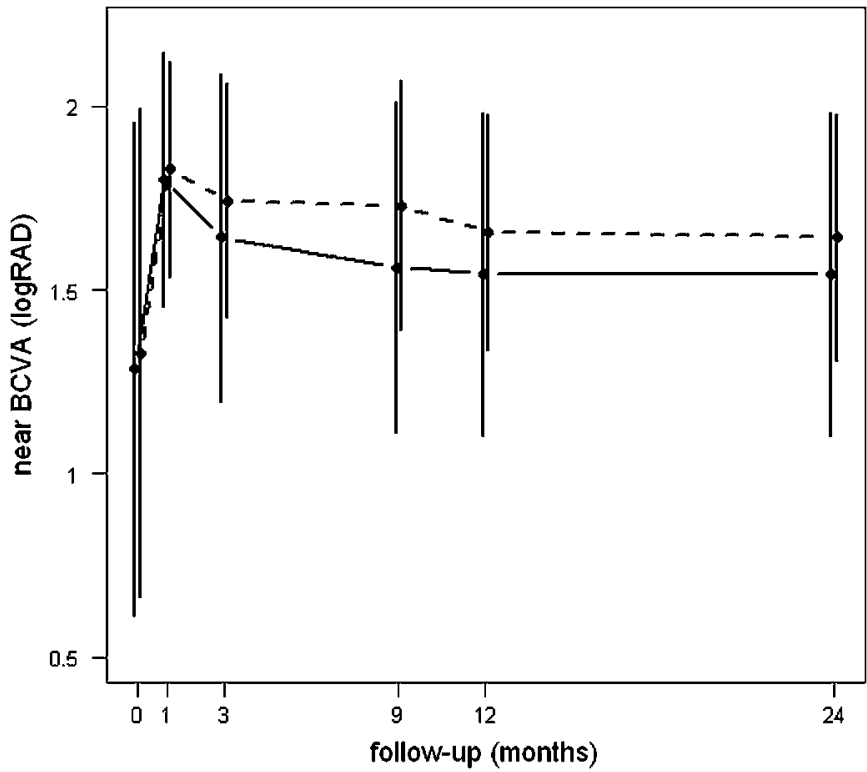

Figure 2 Mean values (points) and standard deviations (vertical lines) of the near best corrected visual acuity (BCVA) measurements using Radner reading charts (logarithm of reading acuity determination (logRAD)) over time (baseline, and at 1, 3, 9, 12 and 24 months) are presented for treatment group 1 (retinal pigment epithelium (RPE)choroid sheet transplantation, solid line) and treatment group 2 (RPE-cell suspension transplantation, dashed line).

abnormal overlying retina with a missing clear outer retinal zone. Details are presented in figure 5.

Microperimetry at 24 months demonstrated retinal fixation over the graft in six patients in group 1, and in two patients in group 2. Fixation was graded as relatively unstable in two patients in group 1, and unstable in four patients in group 1 and in two patients in group 2 .

\section{DISCUSSION}

This randomised study evaluated the outcomes of two RPE transplantation techniques in patients with advanced neovascular AMD who were unsuitable for or were non-responders to other forms of treatment. Transplantation of RPE-choroid sheet and RPE-cell suspension enabled the maintenance of distance BCVA or in the best cases the restoration of foveal function. However, reading ability was rarely restored or improved.

Baseline demographics were comparable between both groups in our series $(p>0.05)$. Contrary to other reports ${ }^{15}{ }^{16}$ the size of the lesion/haemorrhage and the type of the CNV were not significantly related to the visual outcome in both groups in our study ( $p>0.05)$.

The postoperative complication rates were comparable between both treatment groups $(p=0.56)$. Higher surgeryrelated complication rates were reported after RPE-choroid sheet transplantation. ${ }^{7} 8$ 13-15 $17-19$ No recurrence of CNV was detected angiographically in any of our patients. This is in accordance with two studies on RPE-choroid sheet cases in geographic atrophy and neovascular AMD. ${ }^{17} 18$ One comparative study on neovascular $\mathrm{AMD}$ reported no recurrence in the graft group, but recurrence in the macular translocation group (four out of 12 patients), ${ }^{16}$ and another study found recurrence of CNV in 11 out of 84 RPE-choroid sheet cases. ${ }^{15}$

The functional outcome was comparable between both groups in our series and quite similar to the results reported in the literature..$^{17-19}$ Duration of vision loss has been reported 

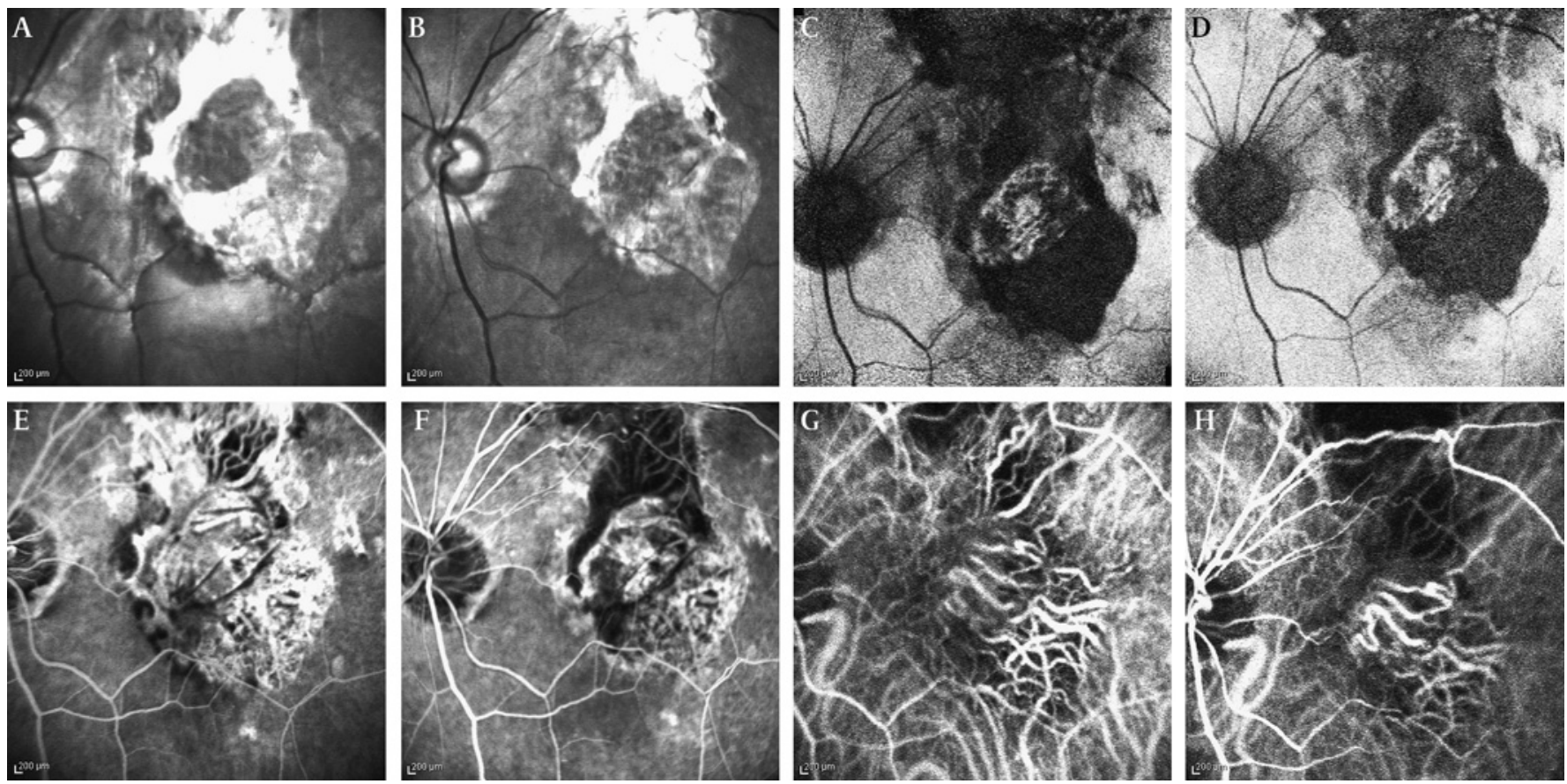

Figure 3 Images at 12 months $(A, C, E, G)$ and 24 months $(B, D, F, H)$ of a patient who underwent retinal pigment epithelium (RPE)-choroid sheet transplantation on his left eye for a large subretinal haemorrhage not responding to photodynamic therapy (PDT) treatment. Twenty-four months after surgery, he presented with a significant improvement in distance BCVA and a regain in reading vision. The graft is clearly visible as a dark area surrounded by the bright atrophy in red-free photographs $(A, B)$, and as a hyperfluorescent area within the hypofluorescent atrophy in autofluorescence $(C, D)$. Revascularisation of the graft is shown in fluorescein $(E, F)$ and indocyanine green angiography $(G, H)$. These findings remained stable during follow-up.
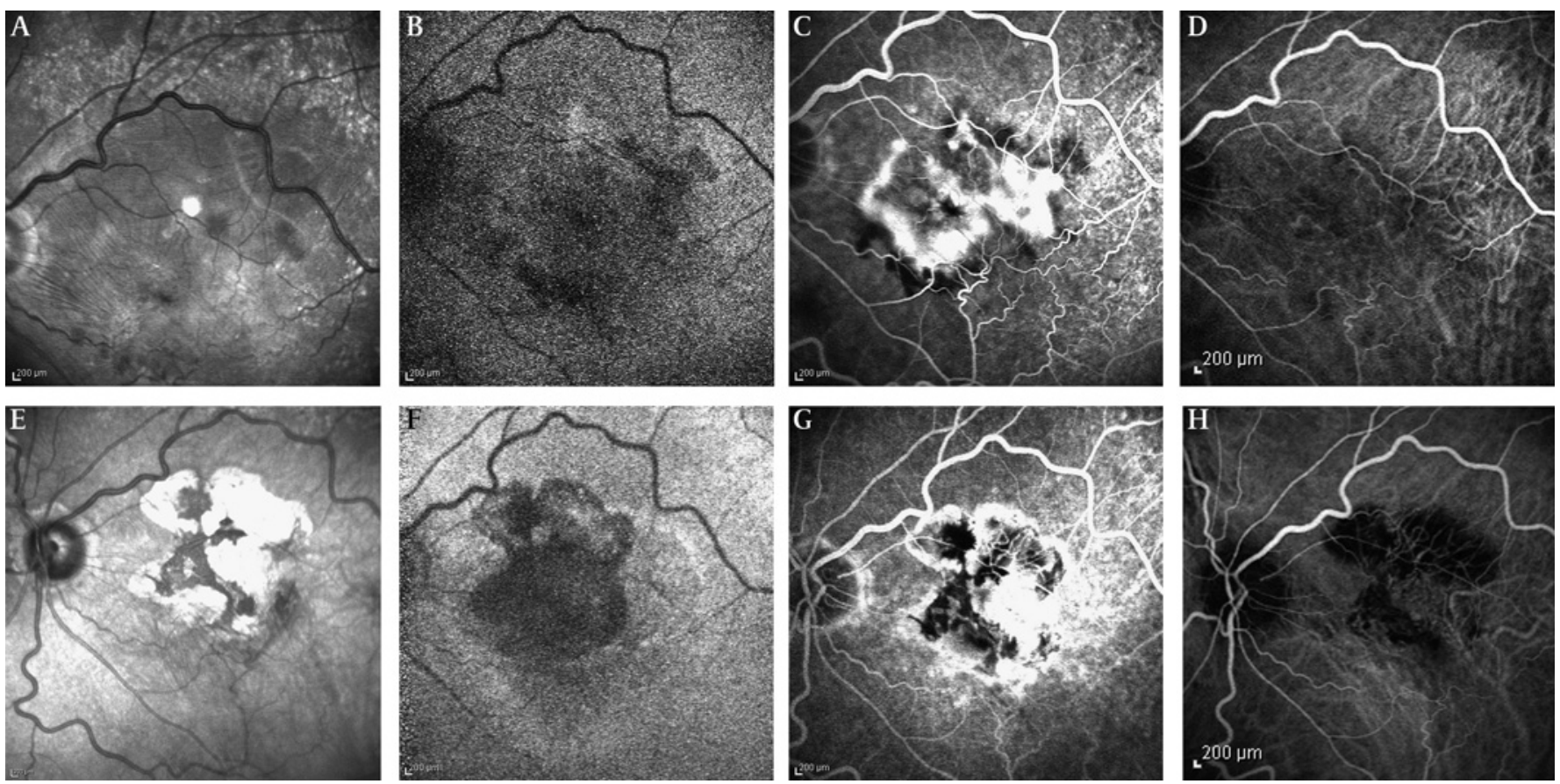

Figure 4 Red-free photographs (RF), autofluorescence (AF), and fluorescein and indocyanine green angiography (FAG, ICG) images of a patient with a diagnosis of choroid neovascularisation (CNV) and pigment epithelium detachment in his left eye. Twenty-four months after retinal pigment epithelium (RPE) cell-suspension transplantation, his distance best corrected visual acuity (BCVA) remained stable, but he did not recover any reading vision. Baseline images show a partially fibrotic lesion with retinal folds, surrounded by haemorrhage (corresponding to blockage in FAG) (RF (A)), inhomogeneous hypofluorescence in the area of the lesion (AF (B)), blocked fluorescein in the area of subretinal haemorrhage, hyperfluorescence and leakage corresponding to the CNV (FAG (C)), and dilated vessels and choriocapillaris defects within the lesion (ICG (D)). Images at 24 months show areas of hyperpigmentation (dark) and atrophy (bright) (RF (E)), hypofluorescence with isles of hyperfluorescence (AF (F)), blocked fluorescein in the area of hyperpigmentation and hyperfluorescence in the area of the RPE atrophy (FAG (G)) and choriocapillaris defects in a normal choroidal vasculature (ICG $(\mathrm{H}))$. 
Figure 5 Time-domain and spectraldomain optical coherence tomography (TD- and SD-OCT) images at 24 months after retinal pigment epithelium (RPE)choroid sheet transplantation $(A, B)$ and RPE cell-suspension transplantation (C, D). In both treatment groups, an increased permeability of light into the tissue is visible in areas of RPE atrophy. This increased permeability is absent in the area of the graft. No signs of fluid leakage are visible in both groups. The retinal thickness (RT) anterior to the graft seems normal (except for a small area) compared with subnormal in the area of the cell suspension. The outer retinal zone was absent in an abnormal retina overlying the transplant in both groups. These cell irregularities and the retinal and subretinal layers are more clearly shown in the SD OCT scans.
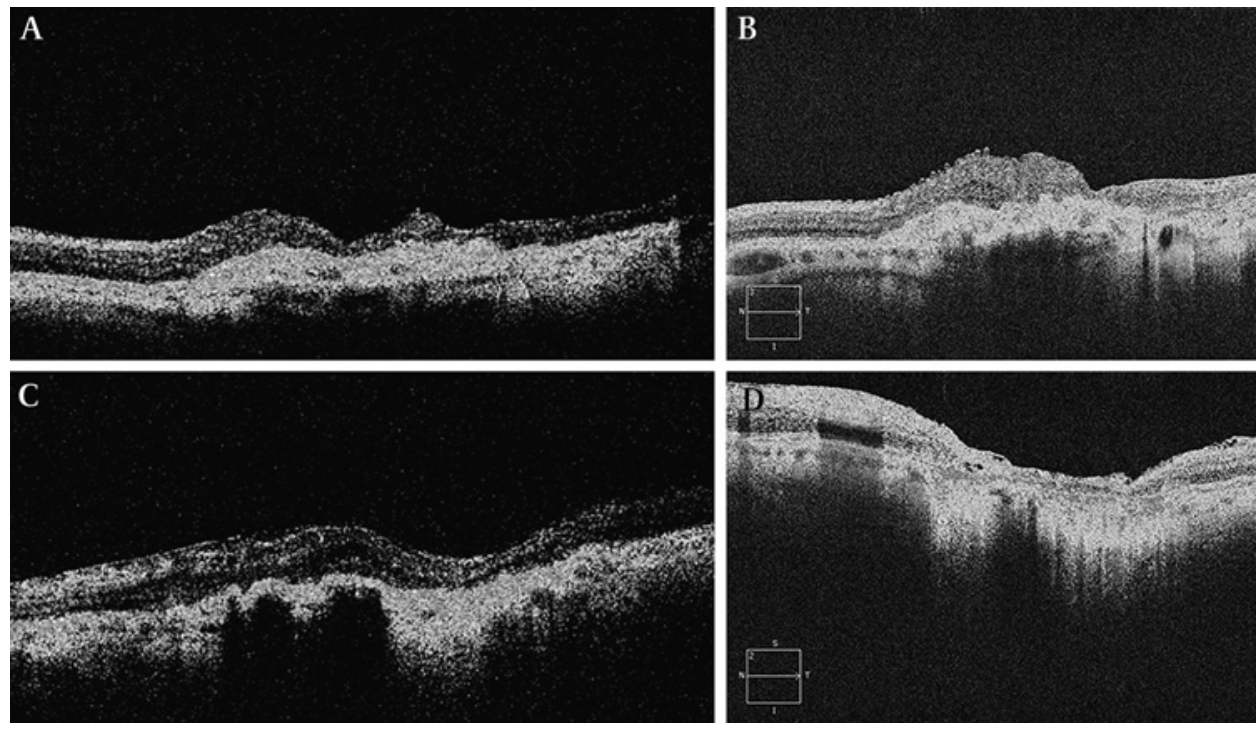

to be a significant predictor for the functional outcome after RPE-choroid sheet transplantation and macular translocation. ${ }^{15-17}$ Half of the patients included in our study were non-responders to previous treatment, and five patients out of these seven non-responders underwent RPE-choroid sheet transplantation. This longstanding vision loss with already impaired function of the photoreceptors and the neurosensory retina may be another reason for the less favourable visual results in group 1 . As our study started in 2004, we were not able to include microperimetry, which would have provided additional information on retinal function in our baseline and early postoperative follow-up. However, eight patients had retinal fixation over the graft at 24 months, but fixation was graded relatively unstable in two patients after RPE-choroid sheet transplantation and unstable in six patients after both RPE transplantation techniques. Other studies reported better results on fixation stability after RPE-choroid sheet transplantation. ${ }^{15} 19$

Transplantation of RPE-choroid sheet provides an organised cell layer over its own basal lamina, whereas transplantation of RPEcell suspension has the disadvantage that a limited amount of cells is distributed over a damaged basal lamina with an uncertain fate. ${ }^{20} 21$ This implies that uneventful transplantation of RPEchoroid sheet, being a better cell source than RPE-cell suspension, may offer a greater chance of regaining normal retinal structures and thus visual function. In addition, our previously published studies on RPE-cell suspension have shown that patients with smaller membranes had a greater chance of regaining vision compared with patients with large pathologies. ${ }^{814}$ Nevertheless, the RPE-choroid sheet group consisting of patients with large pathologies having uneventful transplantation did not show a significantly better outcome. Further evaluation offered two explanations for this limited visual outcome. (1) The RPE-choroid sheets that reached the edge of normal RPE on one side started building RPE bridges from this side. These grafts showed better maintenance in colour and autofluorescence over time. Although two patients presented with regain in vision after surgery, only one of these patients showed a continuous visual recovery over more than 18 months. Five of our RPE-choroid sheet patients presented with grafts not close enough to the normal RPE to create these bridges. In addition, they showed a decrease of the size of the graft, which were surrounded by fibrotic tissue. (2) The RPE-choroid sheets looked regular and well organised on TDOCT. However, on SD-OCT irregularities in the cell layers within the graft were visualised. These irregularities suspicious of scar formation or increased gliosis may also explain the inadequate visual recovery. In addition, we found an absent clear outer nuclear and photoreceptor layer as previously described by MacLaren et al, which they interpreted as photoreceptor loss over the graft. $^{22}$

Our study has some limitations. Although there has been no previous report evaluating the outcome after transplantation of RPE-choroid sheet and RPE-cell suspension, the sample size in the present study is relatively small. There may be limited power to detect outcome differences and this may lead to insufficiency of statistical analysis. The 24 months of follow-up seems to be appropriate for evaluating these different transplantation techniques, although anatomical as well as visual outcomes may still change even 3 to 6 years after surgery. ${ }^{22}$

In conclusion, transplantation of RPE offers an alternative approach in advanced AMD, and is also suitable for geographic $\mathrm{AMD}$ and other degenerative retinal diseases. However, the functional results with RPE transplantation techniques do not approach the levels of outcome seen with anti-VEGF treatment. Although complication rates after transplantation of RPE seem to be greater, long-term effects of repeated intravitreal application are still unknown. ${ }^{23} 24$ Refinement of surgical technique to allow for an adequate graft size, improvement of the quality of RPE and Bruch's membrane (rejuvenation, gene therapy, prosthetic Bruch's membrane, stem cells) and combination therapies might be future options to improve outcome after transplantation of RPE. Refined indications for treatment (eg, patients with fresh RPE rips involving the fovea or 'non-responders' at an earlier time) should be evaluated in multicentre trials.

Competing interests None declared.

Patient consent Obtained.

Ethics approval This study was conducted with the approval of the Ethics Committee of the City of Vienna and complied with the Declaration of Helsinki.

Contributors Design of the study (CIF-R, SB), conduct of the study (CIF-R, IK, CG, BP, WD, AG, SB), collection and management (CIF-R), analysis (CIF-R, IK, AG), interpretation (CIF-R, IK, AG, SB), preparation (CIF-R, IK, AG, SB), review and final approval (CIF-R, IK, AG, SB).

Provenance and peer review Not commissioned; externally peer reviewed.

\section{REFERENCES}

1. Del Priore LV, Tezel TH, Kaplan HJ. Maculoplastyfor age-related macular degeneration: reengineering Burch's membrane and the human macula. Prog Retin Eye Res 2006;25:539-62. 
2. Flood MT, Gouras P, Kjeldbye H. Growth characteristics and ultrastructure of human retinal pigment epithelium in vitro. Invest Ophthalmol Vis Sci 1980;19:1309-20.

3. Lopez R, Gouras P, Brittis M, et al. Transplantation of cultured rabbit retinal epithelium to rabbit retina using a closed-eye method. Invest Ophthalmol Vis Sci 1987;19:1131-7.

4. Gouras $\mathbf{P}$, Algvere P. Retinal cell transplantation in the macula: new techniques. Vision Res 1996;36:4121-5.

5. Aisenbrey S, Lafaut BA, Szurman P, et al. Iris pigment epithelial translocation in the treatment of exudative macular degeneration: a 3-year follow-up. Arch Ophthalmol 2006;124:183-8.

6. Chai $\mathbf{H}$, Shin MC, Tezel TH, et al. Use of iris pigment epithelium to replace retinal pigment epithelium in age-related macular degeneration: a gene expression analysis. Arch Ophthalmol 2006;124:1276-85

7. van Meurs JC, ter Averst E, Hofland LJ, et al. Autologous peripheral retinal pigment epithelium translocation in patients with subfoveal neovascular membranes. Br J Ophthalmol 2004;88:110-13.

8. Binder S, Krebs I, Hilgers RD, et al. Outcome of transplantation of autologous retinal pigment epithelium in age-related macular degeneration: a prospective trial. Invest Ophthalmol Vis Sci 2004;45:4151-60.

9. Stanga PE, Kychenthal A, Fitzke FW et al. Retinal pigment epithelium translocation after choroidal neovascular membrane removal in age-related macular-degeneration. Ophthalmology 2002;109:1492-8.

10. Tezel TH, Del Priore LV, Berger AS, et al. Adult retinal pigment epithelia transplantation in exsudative age-related macular degeneration. Am J Ophthalmol 2007:143:584-95.

11. Binder S, Stanzel BV, Krebs I, et al. Transplantation of RPE in AMD. Prog Retin Eye Res 2007;26:516-54.

12. da Cruz L, Chen FK, Ahmado A, et al. RPE transplantation and its role in retinal disease. Prog Retin Eye Res 2007;26:598-635.

13. van Meurs JC, Van den Biesen PR. Autologous retinal pigment epithelium and choroids translocation in patients with exudative age-related macular degeneration: short term follow-up. Am J Ophthalmol 2003;1136:688-95.
14. Binder S, Stolba U, Krebs I, et al. Transplantation of autologous retinal pigment epithelium in eyes with foveal neovascularization resulting from age-related macula degeneration: a pilot study. Am J Ophthalmol 2002;133:215-25.

15. Maaijwee K, Heimann $\mathrm{H}$, Missotten $\mathrm{T}$, et al. Retinal pigment epithelium and choroids translocation in patients with exudative age-related macular-degeneration: long term results. Graefes Arch Clin Exp Ophthalmol 2007;245:1681-9.

16. Chen FK, Patel PJ, Uppal GS, et al. A comparison of macular translocation with patch graft in neovascular age-related macular degeneration. Invest Ophthalmol Vis Sci 2009;50:1848-55.

17. Joussen AM, Joeres S, Fawzy N, et al. Autologous translocation of the choroids and retinal pigment epithelium in patients with geographic atrophy. Ophthalmology 2007:114:551-60.

18. MacLaren RE, Uppal GS, Balaggan KS, et al. Autologous transplantation of the retinal pigment epithelium and choroids in the treatment of neovascular age-related macular degeneration. Ophthalmology 2007:114:561-570.

19. Treumer F, Bunse A, Klatt C, et al. Autologous retinal pigment epithelium-choroid sheet transplantation in age related macular degeneration: morphological and functional results. $\mathrm{Br} J$ Ophthalmol 2007:91:349-53.

20. Tsukahara I, Ninomiya S, Castellarin A, et al. Early attachment of uncultured retinal pigment epithelium from aged donors onto Bruch's membrane explants. Exp Eye Res 2002; 74:255-66.

21. Zarbin MA. Analysis of retinal pigment epithelium integrin expression and adhesion to aged submacular human Bruch's membrane. Trans Am Ophthalmol Soc 2003; 101:499-520.

22. MacLaren RE, Bird AC, Sathia PJ, et al. Long-term results of submacular surgery combined with macular translocation of the retinal pigment epithelium in neovascula age-related macular degeneration. Ophthalmology 2005;112:2081-7.

23. Ciulla TA, Rosenfeld PJ. Antivascular endothelial growth factor therapy for neovascular age-related macular degeneration. Curr Opin Ophthalmol 2009;20:158-65

24. Jeganathan VS, Verma N. Safety and efficacy of intravitreal anti-VEGF injections fo age-related macular degeneration. Curr Opin Ophthalmol 2009;20:223-5. 